\title{
UTILIZAÇÃO DA PROGRAMAÇÃO LINEAR NA FORMULAÇÃO DE PASTA DE VEGETAIS ENRIQUECIDA COM PLASMA BOVINO E SECAGEM EM LEITO DE JORRO
}

\author{
S. F. ROCHA ${ }^{1 *}$, T. MULLER ${ }^{1}$, C. K. BIERHALS ${ }^{1}$, J. V. O. CASTRO ${ }^{1}$, A. P. Q. LARROSA ${ }^{1}$, L. A. \\ A. PINTO ${ }^{1}$ \\ ${ }^{1}$ Universidade Federal do Rio Grande, Escola de Química e Alimentos \\ *e-mail: silvinharocha_@ hotmail.com
}

\begin{abstract}
RESUMO
O aproveitamento de excedentes do setor agropecuário visa aumentar a disponibilidade de alimentos de alto teor proteico. O objetivo deste trabalho foi utilizar a programação linear para formular uma pasta de vegetais enriquecida de plasma bovino visando à maximização do teor proteico, e avaliação da secagem da pasta em leito de jorro nas características do produto final. A otimização da formulação da pasta de vegetais foi baseada em equações e inequações lineares sob restrições de teor de carboidratos, proteínas, lipídios e valor calórico de acordo com sopas comerciais. Os experimentos de secagem foram realizados em leito de jorro cônico, utilizando vazões de alimentação de 150 e $250 \mathrm{~mL} \mathrm{~h}^{-1}$ e temperaturas do ar de entrada de 90 e $110^{\circ} \mathrm{C}$. Os produtos desidratados foram avaliados quanto a solubilidade protéica, capacidade de retenção de água, índice de solubilidade em água e parâmetros de cor. O tratamento com $150 \mathrm{~mL} \mathrm{~h}^{-1}$ e $90^{\circ} \mathrm{C}$ apresentou os melhores resultados quanto às propriedades funcionais das proteínas. Nessa condição, o produto final apresentou tonalidade amarelo-esverdeada.
\end{abstract}

\section{INTRODUÇÃO}

O desperdício de hortifrutigranjeiros precisa ser revertido para aumentar a disponibilidade de alimentos, pois mais da metade da produção de frutas e verduras é desperdiçada na América Latina. Cerca de 1,4 bilhões de toneladas de vegetais produzidos são descartados antes de sair da propriedade rural, devido ao manuseio inadequado (FAO, 2011).

O segmento frigorífico é responsável por gerar um grande volume de sangue, produto de alto potencial alimentar e poder contaminante. Apesar das excelentes propriedades nutricionais e funcionais do sangue inteiro, existe limitações quanto a sua utilização em formulações alimentícias no Brasil, pois há uma preocupação com as características organolépticas do sangue que causariam uma rejeição dos produtos pelos consumidores (GOULART, 2000). Diante disso, o fracionamento do sangue para a utilização apenas do plasma sanguíneo é uma alternativa para o enriquecimento proteico de novas formulações de produtos.

O uso de vegetais e proteínas de origem animal, na alimentação humana oferecem benefícios à saúde, pois o plasma bovino tem ampla utilização como fonte proteica em produtos alimentícios, devido a suas propriedades funcionais de solubilidade e capacidade de emulsificação, sem alterar as características organolépticas do produto final (ORNELLAS, 2006).

A programação linear tem sido utilizada em formulações de alimentos, através de modelos matemáticos, maximizando lucros 
ou minimizando custos de produção. É possível estabelecer uma mistura ótima de diversas variáveis, segundo uma função linear de efetividade, satisfazendo um conjunto de restrições lineares para estas variáveis (PRADO, 2010).

A secagem de alimentos é comumente utilizada para reduzir o teor de umidade, aumentando o tempo de conservação (MUJUMDAR; HUANG, 2007). Essa crescente demanda requer a otimização das condições da operação de secagem, especialmente a temperatura, com a finalidade de realizar não apenas a eficiência do processo, mas também a qualidade final do produto seco (BANGA et al., 2003). O leito de jorro tem sido empregado para secagem de pastas e suspensões, sendo uma alternativa ao spray dryer, mantendo o mesmo nível de qualidade do produto e baixo custo (OLIVEIRA; FREITAS, 2004). A rápida desidratação obtida neste tipo de secador conserva as características naturais dos alimentos (SHUHAMA et al., 2003).

O objetivo deste trabalho foi utilizar a programação linear para formular uma pasta de vegetais enriquecida de plasma bovino, visando à maximização do teor proteico, e avaliação da secagem da pasta em leito de jorro nas características do produto final.

\section{MATERIAL E MÉTODOS}

\subsection{Matéria-prima}

Para obtenção da pasta de vegetais enriquecida de plasma bovino foram utilizados os seguintes constituintes: cebola branca (Allium cepa L.), tomate (Lycopersicum esculentum), cenoura (Daucus carota L.), couve (Brassica oleracea), batata (Solanum tuberosum), óleo de soja comercial e plasma bovino.

O sangue bovino necessário para obtenção do plasma foi coletado em um abatedouro situado na cidade de Pelotas/RS com as práticas de assepsia devidamente empregadas, para extração do plasma bovino foi feita a centrifugação do sangue a uma rotação de $3500 \times \mathrm{g}$ por $15 \mathrm{~min}$ (LOPES et al., 2004). Os vegetais e o óleo de soja foram adquiridos em feiras livres e nos mercados da cidade do Rio Grande/RS, respectivamente.

\subsection{Formulação da Pasta de Vegetais Enriquecida de Plasma Bovino}

A pasta foi formulada utilizando a programação linear otimizando a maximização do conteúdo proteico, sendo utilizadas as restrições de teores carboidratos, proteínas e lipídios, e valor calórico. A Tabela 1 mostra as restrições utilizadas para formular a pasta.

Tabela 1 - Restrições para formulação da pasta de vegetais enriquecida de plasma bovino, otimizando a maximização do teor proteico.

\begin{tabular}{ccc}
\hline & $\begin{array}{c}\text { Limite } \\
\text { mínimo }\end{array}$ & $\begin{array}{c}\text { Limite } \\
\text { máximo }\end{array}$ \\
\hline Carboidratos & $50 \%$ & $70 \%$ \\
Proteínas & $15 \%$ & $20 \%$ \\
Lipídios & $4 \%$ & $4 \%$ \\
Calorias & $200 \mathrm{kcal}$ & $500 \mathrm{kcal}$ \\
\hline
\end{tabular}

Os valores das restrições apresentados na Tabela 1 foram baseados em uma formulação de sopa desidratada comercial (IBGE, 1999). Com o objetivo de obter um produto de elevado valor proteico, foi necessário que os valores estabelecidos desta formulação fossem alterados. Segundo Brasil (1998a), para alimentos enriquecidos ou fortificados é permitido o enriquecimento ou fortificação desde que $100 \mathrm{~mL}$ ou $100 \mathrm{~g}$ do produto, pronto para consumo, forneçam no mínimo $15 \%$ da ingestão diária recomendada (IDR) de referência, no caso de líquidos ou alimentos pastosos.

Sabendo que a IDR de proteína é de 50 g para um adulto (BRASIL, 1998b) definiu-se que a pasta deveria conter, no mínimo $15 \%$ de proteína. Portanto, foi estabelecido o intervalo de 15 a $20 \%$ de proteína. Para os limites 
mínimo e máximo de lipídios foram fixados a $4 \%$, devido a estudos anteriores apresentarem bons resultados com relação à escoabilidade do leito de jorro (ROCHA et al., 2014).

O aplicativo Microsoft Solver Excel permitiu fazer a otimização utilizando o teor proteico como função objetivo. Essa função é uma equação linear que relaciona todos os constituintes com seu respectivo teor proteico segundo a Equação 1.

$$
P_{T}=\sum_{i=1}^{n} m_{i} \cdot P_{i}
$$

As funções de restrição (Equações 2 a 12) são equações e inequações que limitam a função objetivo. A Equação 2 relaciona as massas de cada constituinte em base seca.

$C_{B S}=m_{1}+m_{2}+m_{3}+m_{4}+m_{5}+m_{6}+m_{7}$

As Inequações 3 e 4 limitam o teor de carboidratos $(C)$; as Inequações 5 e 6 limitam o teor de proteínas $(P)$; as Inequações 7 e 8 limitam o teor de lipídios $(L)$; as Inequações 9 e 10 limitam o valor calórico $(V c)$; as Inequações 11 e 12 restringem a possibilidade de valores negativos para quantidade de massa e dos constituintes com quantidade máxima estabelecida, respectivamente.

$$
\begin{aligned}
& \sum_{i=1}^{i=7} m_{i} c_{i} \geq C_{1} \\
& \sum_{i=1}^{i=7} m_{i} c_{i} \leq C_{2} \\
& \sum_{i=1}^{i=7} m_{i} p_{i} \geq P_{1} \\
& \sum_{i=1}^{i=7} m_{i} p_{i} \leq P_{2} \\
& \sum_{i=1}^{i=7} m_{i} l_{i} \geq L_{1}
\end{aligned}
$$

$$
\begin{aligned}
& \sum_{i=1}^{i=7} m_{i} l_{i} \leq L_{2} \\
& \sum_{i=1}^{i=7} m_{i} v c_{i} \geq V c_{1} \\
& \sum_{i=1}^{i=7} m_{i} v c_{i} \leq V c_{2} \\
& m_{i \geq 0} \\
& m_{i} \leq m
\end{aligned}
$$

\subsection{Obtenção da Pasta de Vegetais enriquecida de plasma bovino}

Para obter a pasta, foram realizadas as etapas descritas na Figura 1.

Figura 1 - Fluxograma do processamento da pasta de vegetais enriquecida de plasma bovino.

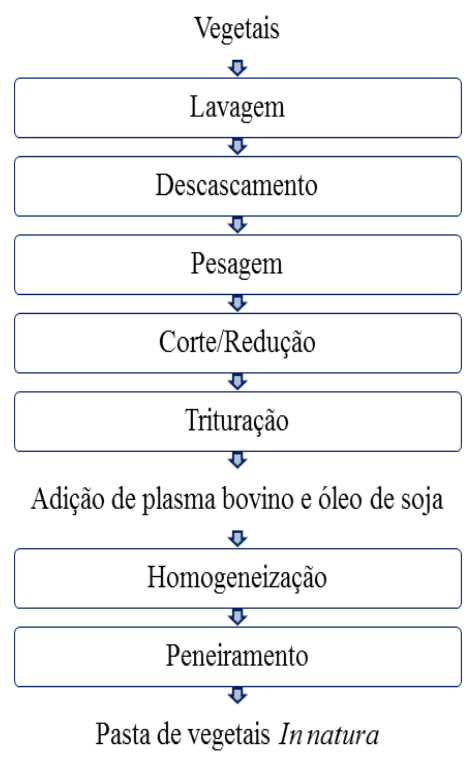

Fonte: Autor (2015).

\subsection{Secagem em Leito de Jorro}

A secagem foi realizada em célula de geometria cônica, com o diâmetro de $17,5 \mathrm{~cm}$, com extremidades inferior e superior de vidro com ângulo de $60^{\circ}$ e altura de $15 \mathrm{~cm}$ cada, com diâmetro de entrada de $2,9 \mathrm{~cm}$ e a relação 
(Dc/ Di) igual a 6,0. O leito de inertes foi composto por $0,50 \mathrm{~kg}$ de partículas de polietileno com diâmetro médio de $3,2 \mathrm{~mm}$, esfericidade 0,7 e densidade de $0,96 \mathrm{~g} . \mathrm{cm}^{-3}$.

O fluido utilizado na secagem foi ar aquecido por um conjunto de três resistências (800 W cada). A vazão de ar foi determinada por placa de orifício acoplada a um manômetro de tubo em "U", e as medidas de temperaturas foram feitas por termopares cobre-constantan. Foram fixadas a pressão de atomização em $200 \mathrm{kPa}$ absoluta e a taxa de circulação de sólidos de $100 \%$ acima da velocidade de jorro mínimo.

A pasta teve a sua concentração inicial ajustada com água para $5 \%(\mathrm{~m} / \mathrm{m})$ de sólidos, e foi alimentada no leito com sistema semicontínuo com auxílio de uma seringa com capacidade de $50 \mathrm{~mL}$, em intervalos de 1 a 2 min. O regime da operação foi monitorado pelas medidas das temperaturas do ar de entrada e saída da célula de secagem das temperaturas de bulbo seco e de bulbo úmido do ar na saída do ciclone a cada 2 min até atingir $30 \mathrm{~min}$, e após a cada 5 min durante a operação. O tempo total de operação para cada experimento de secagem foi de $6 \mathrm{~h}$, e o pó seco foi recolhido em um recipiente de vidro acoplado ao ciclone. O esquema do equipamento experimental de leito de jorro está apresentado na Figura 2.

Figura 2 - Equipamento de Secagem em Leito de Jorro.

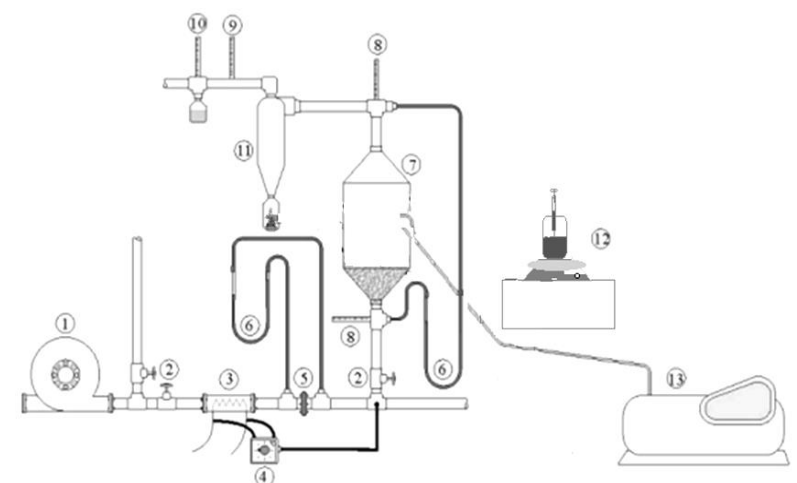

Fonte: Larrosa (2012).
Onde: (1) soprador radial; (2) válvulas de regulagem de vazão de ar; (3) sistema de aquecimento do ar; (4) controlador de temperatura; (5) placa de orifício; (6) manômetro de tubo em U; (7) célula de secagem; (8) termopares de entrada e saída da célula de secagem; (9) termopar de bulbo seco; (10) termopar de bulbo úmido; (11) ciclone tipo Lapple; (12) reservatório da pasta; (13) compressor de ar.

\subsection{Análise Estatística}

Os experimentos de secagem tiveram dois fatores de estudo (vazão de alimentação da pasta e temperatura do ar de entrada) em dois níveis de operação. A Tabela 2 mostra os fatores de estudo com seus respectivos níveis.

Tabela 2 - Fatores e respectivos níveis de estudo dos experimentos de secagem de pasta de vegetais enriquecida de plasma bovino.

\begin{tabular}{ccc}
\hline $\begin{array}{c}\text { Fator de } \\
\text { Estudo }\end{array}$ & Nível Inferior & Nível Superior \\
\hline $\begin{array}{c}\text { Vazão de } \\
\text { alimentação } \\
\text { da pasta }(\mathrm{mL}\end{array}$ & 150 & 250 \\
$\left.\mathrm{~h}^{-1}\right)$ & & \\
$\begin{array}{c}\text { Temperatura } \\
\text { do ar de }\end{array}$ & 90 & 110 \\
entrada $\left({ }^{\circ} \mathrm{C}\right)$ & & \\
\hline
\end{tabular}

Os resultados foram analisados através do Teste de Tukey ao nível de $95 \%$ de confiança $(\mathrm{p}<0,05)$.

\subsection{Metodologia analítica}

2.6.1 Solubilibidade Proteica em meio aquoso

A solubilidade proteica foi determinada segundo a metodologia descrita por Morr et al. (1985). Para a quantificação da solubilidade adicionou-se aproximadamente 2,5 g de amostra em $50 \mathrm{~mL}$ de água destilada, a seguir levou-se ao agitador magnético por 15 min e posterior centrifugação a $3500 \mathrm{rpm}$ por $15 \mathrm{~min}$. Após a centrifugação, filtrou-se o conteúdo dos tubos, e procedeu-se a determinação de proteína do sobrenadante. $\mathrm{O}$ índice de proteína solúvel foi determinado sobre o conteúdo total de proteína das amostras como mostra a Equação 13.

$$
P S=\frac{A \times 50}{W \times \frac{S}{100}} \times 100
$$


2.6.2 Capacidade de Retenção de Água (CRA) e Índice de Solubilidade em Água (ISA)

A capacidade de retenção de água foi quantificada segundo adaptações de Anderson et al., (1969). Para determinação da $C R A$ e $I S A$, elaborou-se uma mistura contendo $2,5 \mathrm{~g}$ de amostra e $30 \mathrm{~mL}$ de água, depois de mantida sob agitação por $30 \mathrm{~min} e$ posteriormente centrifugada a $5000 \times$ g por 15 min na temperatura de $24{ }^{\circ} \mathrm{C}$. O sedimento foi pesado e, no sobrenadante determinado resíduo seco (sólido solúveis) após evaporação. Os índices foram então calculados de acordo com as Equações $14 \mathrm{e}$ 15 .

$$
\begin{gathered}
C R A=\frac{M_{r c}}{\left(M_{a}-M_{r e}\right)} \\
I S A=\frac{M_{r e} \times 100}{M_{a}}
\end{gathered}
$$

\subsubsection{Cor}

Os parâmetros de cor da pasta in natura e $\mathrm{o}$ produto desidratado foram avaliados utilizando-se um colorímetro Minolta (CR-300, Osaka, Japão), por meio do diagrama tridimensional de cores $\left(L^{*} a * b^{*}\right)$, O índice de saturação $\left(C^{*}\right)$ das amostras foi determinado pela Equação 16, o ângulo Hue $(\alpha)$ conforme a Equação 17 e a variação da cor $(\Delta E)$ foi determinada pela Equação 18. O ângulo Hue é o valor em graus correspondente ao diagrama tridimensional de cores (SRINVASA et al., 2004). A variação da cor relaciona as diferenças de cor entre a amostra in natura e a amostra desidratada.

$$
\begin{aligned}
& C^{*}=\sqrt{\left(a^{*}\right)^{2}+\left(b^{*}\right)^{2}} \\
& \alpha=\tan ^{-1}\left(\left(\frac{b^{*}}{a^{*}}\right)\right) \\
& \Delta E=\sqrt{\left(\Delta L^{*}\right)^{2}+\left(\Delta a^{*}\right)^{2}+\left(\Delta b^{*}\right)^{2}}
\end{aligned}
$$

\section{RESULTADOS E DISCUSSÃO}

A Tabela 3 apresenta formulação otimizada pela programação linear para maximização de proteínas da pasta in natura.

Tabela 3: Quantidades dos constituintes otimizados para formulação da pasta de vegetais enriquecida com plasma bovino.

\begin{tabular}{lc}
\hline Constituintes & Quantidade $(\mathrm{g})$ \\
\hline Tomate & 361,3 \\
Couve & 278,5 \\
Batata & 130,7 \\
Cebola & 69,9 \\
Cenoura & 18,0 \\
Óleo de soja & 0,82 \\
Plasma bovino & 40,8 \\
\hline
\end{tabular}

Fonte: Autor (2015).

Observa-se na Tabela 3 que o constituinte que apresentou maior quantidade foi o tomate, isso ocorreu devido ao fato deste vegetal possuir alto teor de carboidratos. Embora a função objetivo da programação linear tenha sido a maximização de proteínas, dentre as restrições utilizadas foi estabelecida uma proporção maior para os carboidratos (50-70\%). Portanto, a programação linear obteve uma combinação dentre as restrições estabelecidas juntamente com a função objetivo, determinando as quantidades de cada constituinte.

A Tabela 4 mostra o resultado da otimização das restrições utilizadas na formulação da pasta de vegetais enriquecida de plasma bovino pela programação linear.

Tabela 4: Restrições otimizadas no teor de nutrientes da pasta de vegetais enriquecida com plasma bovino.

Programação Linear

\begin{tabular}{cc} 
& (B.S.) \\
\hline Carboidratos & $50,0 \%$ \\
Proteínas & $20,0 \%$ \\
Lipídios & $4,0 \%$ \\
\hline
\end{tabular}




Valor Calórico $\quad 316,0 \mathrm{kcal}$

Fonte: Autor (2015).

De acordo com a Tabela 4, os valores otimizados estão entre os limites estabelecidos nas restrições, mostrando que a programação linear foi adequada para a formulação da pasta de vegetais enriquecida de plasma bovino através da maximização do teor proteico. Tendo o teor proteico atingido o valor máximo estabelecido de $20 \%$, obtendose um produto enriquecido.

A Tabela 5 apresenta a avaliação dos produtos desidratados e in natura quanto à solubilidade proteica, capacidade de retenção de água $(C R A)$ e índice de solubilidade em água (ISA).

Tabela 5: Solubilidade proteica, CRA e ISA dos produtos desidratados e in natura.

\begin{tabular}{cccc}
\hline Tratamentos & $\begin{array}{c}\text { Solubilidade } \\
\text { Proteica } \\
(\%)^{*}\end{array}$ & $\begin{array}{c}\text { CRA }(\mathrm{g} \\
\left.\mathrm{g}^{-1}\right)^{*}\end{array}$ & $\begin{array}{c}\text { ISA } \\
(\%)^{*}\end{array}$ \\
\hline$(1)$ & $51,8 \pm 0,5^{\mathrm{a}}$ & $5,8 \pm 0,2^{\mathrm{a}}$ & $27,9 \pm 0,6^{\mathrm{a}}$ \\
(2) & $49,8 \pm 1,0^{\mathrm{b}}$ & $4,9 \pm 0,5^{\mathrm{b}}$ & $26,6 \pm 0,5^{\mathrm{b}}$ \\
(3) & $51,5 \pm 0,5^{\mathrm{a}}$ & $5,8 \pm 0,1^{\mathrm{a}}$ & $16,7 \pm 1,6^{\mathrm{c}}$ \\
(4) & $45,8 \pm 1,0^{\mathrm{c}}$ & $4,9 \pm 0,4^{\mathrm{b}}$ & $24,8 \pm 0,5^{\mathrm{d}}$
\end{tabular}

In natura $\quad 35,5 \pm 0,9^{\mathrm{d}}$

Fonte: Autor (2015).

*Valor médio \pm erro médio $(\mathrm{n}=2)$. Letras diferentes na mesma coluna apresentam diferença significativa $(\mathrm{p}<0,05)$.

Legenda: $C R A=$ capacidade de retenção de água, $I S A=$ índice de solubilidade em água. (1) $150 \mathrm{~mL} \mathrm{~h}^{-1}$ e $90^{\circ} \mathrm{C}$, (2) $150 \mathrm{~mL} \mathrm{~h}^{-1} \mathrm{e} 110^{\circ} \mathrm{C}$, (3) $250 \mathrm{~mL} \mathrm{~h}^{-1}$ e $90^{\circ} \mathrm{C}$, (4) $250 \mathrm{~mL} \mathrm{~h}^{-1} \mathrm{e}$ $110^{\circ} \mathrm{C}$.

Através da Tabela 5, pode-se observar que a solubilidade proteica apresentou uma diferença significativa ao nível de $95 \%$ de significância $(\mathrm{p}<0,05)$ para os tratamentos em relação à amostra in natura. Observa-se também, que os tratamentos a $90^{\circ} \mathrm{C}$ não apresentaram diferença significativa $(p>0,05)$ independente da vazão de alimentação. Em relação à amostra in natura, os experimentos de secagem proporcionaram um aumento da solubilidade das proteínas em meio aquoso. Segundo Sgarbieri (1996), a solubilidade proteica tem a tendência a aumentar em uma faixa de temperatura de $40-50^{\circ} \mathrm{C}$, e acima desta tende a diminuir devido a desnaturação. Como a temperatura do material no leito de jorro estava um pouco acima da temperatura de bulbo úmido do ar de entrada, e os experimentos foram realizados com temperatura de bulbo úmido em torno de $37 \pm 1^{\circ} \mathrm{C}$, portanto, obteve-se o aumento da solubilidade em relação à pasta in natura.

A capacidade de retenção de água apresentou valores de 4,9 a $5,8 \mathrm{~g} . \mathrm{g}^{-1}$, sendo que os melhores resultados foram obtidos na menor temperatura do ar de entrada $\left(90^{\circ} \mathrm{C}\right)$. Isso ocorreu devido ao elevado número de proteínas presentes no plasma, que quando exposto a temperaturas moderadas dentro da célula são capazes de desenvolver algumas funções, e não sofrerem desnaturação total. A fração proteica do plasma bovino contém mais de cem proteínas diferentes, sendo que as principais são albumina sérica, $\alpha$-globulina, $\beta$-globulina, $\gamma$-globulina e fibrinogénio (CRISTAS, 2012). Estas proteínas possuem boas características gelificantes, emulsificantes e de solubilidade que as tornam aptas para utilização no processamento de produtos cárneos. Além disso, as globulinas e albuminas são altamente solúveis e bastante hidrofílicas, o que lhes conferem altos índices de retenção de água (XIONG, 2009).

Em relação ao índice de solubilidade em água (ISA), observa-se que todos os experimentos apresentaram diferença significativa ao nível de $95 \%(\mathrm{p}<0,05)$. Além disso, nota-se que o experimento $\mathrm{n}^{\circ} 3(250 \mathrm{~mL}$ $\mathrm{h}^{-1}$ e $90{ }^{\circ} \mathrm{C}$ ) apresentou menor índice de solubilidade em água. Este comportamento pode ser explicado pela menor temperatura de saída do ar e, consequentemente, maior tempo 
de residência do produto dentro da célula de secagem, reduzindo a capacidade dos componentes solúveis oriundos dos vegetais (amido, pectina) e do plasma (proteínas) de se hidratarem com a água.

A Tabela 6 apresenta os resultados obtidos para luminosidade $\left(L^{*}\right)$, cromaticidades $\left(a^{*}, b^{*}\right)$ para a pasta in natura e produtos desidratados.

Tabela 6: Medidas de luminosidade $\left(L^{*}\right)$ e cromaticidades $\left(a^{*}, b^{*}\right)$ da pasta de vegetais in natura e seca em leito de jorro.

\begin{tabular}{cccc}
\hline Tratamentos & $L^{*}$ & $a^{*}$ & $b^{*}$ \\
\hline$(1)$ & $46,5 \pm 0,6^{\mathrm{a}}$ & $-1,4 \pm 0,6^{\mathrm{a}}$ & $33,1 \pm 0,1^{\mathrm{a}}$ \\
$(2)$ & $52,5 \pm 0,8^{\mathrm{b}}$ & $-1,3 \pm 0,2^{\mathrm{b}}$ & $30,8 \pm 0,2^{\mathrm{b}}$ \\
$(3)$ & $44,4 \pm 1,3^{\mathrm{c}}$ & $-1,8 \pm 0,1^{\mathrm{c}}$ & $34,2 \pm 0,4^{\mathrm{c}}$ \\
$(4)$ & $42,5 \pm 1,2^{\mathrm{d}}$ & $-1,7 \pm 0,2^{\mathrm{d}}$ & $29,6 \pm 0,7^{\mathrm{d}}$ \\
\hline In natura & $30,9 \pm 0,4^{\mathrm{e}}$ & $-5,3 \pm 0,1^{\mathrm{e}}$ & $17,3 \pm 0,1^{\mathrm{e}}$ \\
\hline
\end{tabular}

Fonte: Autor (2015).

Legenda: (1) $150 \mathrm{~mL} \mathrm{~h}^{-1}$ e $90^{\circ} \mathrm{C}$, (2) $150 \mathrm{~mL} \mathrm{~h}^{-1}$ e $110^{\circ} \mathrm{C}$, (3) $250 \mathrm{~mL} \mathrm{~h}^{-1}$ e $90^{\circ} \mathrm{C}$, (4) $250 \mathrm{~mL} \mathrm{~h}^{-1}$ e $110^{\circ} \mathrm{C}$.

Os parâmetros avaliados para a cor do produto final, como luminosidade $\left(L^{*}\right)$, cromaticidades $\left(a^{*}, b^{*}\right)$, mostraram diferença significativa ao nível de $95 \%(\mathrm{p}<0,05)$ nos tratamentos em relação à pasta in natura. A secagem de pastas escuras tende a aumentar a luminosidade do produto final seco (LARROSA, 2012), sendo confirmado pelo aumento dos valores de luminosidade $\left(L^{*}\right)$, o que significa que o produto final ficou mais claro que a pasta de alimentação, visto que quanto mais próximo de 100 mais claro é a amostra.

Quanto aos valores de cromaticidade $a^{*}$, tanto nos tratamentos quanto na pasta in natura foram obtidos valores negativos, mostrando uma tendência da tonalidade verde, sendo que nos tratamentos com a vazão de alimentação de $250 \mathrm{~mL} \mathrm{~h}^{-1}$ apresentaram valores mais próximos da pasta in natura. A tonalidade verde pode ser atribuída à presença de couve na pasta, que apresenta alto teor de clorofila, pigmento responsável pela coloração verde em alguns vegetais. A cromaticidade $b^{*}$ mostrou tendência à tonalidade amarela devido aos valores positivos, no entanto, a operação de secagem proporcionou um aumento desta tonalidade.

A Tabela 7 apresenta os parâmetros de cor (índice de saturação, ângulo Hue e diferença de cor), para a pasta de vegetais in natura e seca em leito de jorro.

Tabela 7: Medidas do índice de saturação $(C)$, ângulo Hue e diferença de cor, para pasta de vegetais in natura e seca em leito de jorro.

\begin{tabular}{cccc}
\hline Tratamentos & $C^{*}$ & $\operatorname{Hue}\left(^{\circ}\right)^{*}$ & $\Delta E^{*}$ \\
\hline$(1)$ & $33,1 \pm 0,6^{\mathrm{a}}$ & $92,4 \pm 0,2^{\mathrm{a}}$ & $22,5 \pm 0,1^{\mathrm{a}}$ \\
$(2)$ & $30,8 \pm 0,8^{\mathrm{b}}$ & $92,4 \pm 0,1^{\mathrm{a}}$ & $27,1 \pm 0,2^{\mathrm{b}}$ \\
(3) & $34,2 \pm 0,3^{\mathrm{c}}$ & $93,1 \pm 0,1^{\mathrm{b}}$ & $21,9 \pm 0,4^{\mathrm{c}}$ \\
(4) & $29,6 \pm 0,2^{\mathrm{d}}$ & $93,3 \pm 0,2^{\mathrm{b}}$ & $17,3 \pm 0,7^{\mathrm{d}}$
\end{tabular}

In natura $\quad 18,1 \pm 0,4^{\mathrm{e}} \quad 176,7 \pm 0,1^{\mathrm{c}}$

Fonte: Autor (2015).

Legenda: (1) $150 \mathrm{~mL} \mathrm{~h}^{-1}$ e $90^{\circ} \mathrm{C}$, (2) $150 \mathrm{~mL} \mathrm{~h}^{-1}$ e $110^{\circ} \mathrm{C}$, (3) $250 \mathrm{~mL} \mathrm{~h}^{-1} \mathrm{e} 90^{\circ} \mathrm{C}$, (4) $250 \mathrm{~mL} \mathrm{~h}^{-1} \mathrm{e} 110^{\circ} \mathrm{C}$.

O índice de saturação $\left(C^{*}\right)$ mostrou um comportamento similar à cromaticidade $b^{*}$, onde $\mathrm{o}$ produto desidratado em todas as condições de operação mostrou um aumento da intensidade da coloração em relação à pasta in natura.

Para os valores de ângulo Hue foram superiores a $90^{\circ}$. Sendo que numa faixa de $90^{\circ}$ a $180^{\circ}$ representa a tonalidade amareloesverdeada, assim para as amostras desidratadas predominou a tonalidade amarelo-esverdeada. Para a amostra in natura é possível afirmar a tonalidade foi verde, pois se encontra próximo de $180^{\circ}$. 
Quanto à diferença de cor $(\Delta E)$, observa-se que a condição que obteve menor alteração em relação à pasta in natura foi no tratamento $\mathrm{n}^{\circ} 4$, que corresponde à maior vazão de alimentação da pasta e maior temperatura de entrada do ar, pois neste experimento obteve-se menores valores de luminosidade e cromaticidade $b$.

Através das avaliações pode-se determinar que a melhor condição de secagem em leito de jorro da pasta de vegetais enriquecida de plasma bovino foi $\mathrm{O}$ tratamento $\mathrm{n}^{\circ} 1\left(150 \mathrm{~mL} \mathrm{~h}^{-1}\right.$ e $\left.90^{\circ} \mathrm{C}\right)$, que obteve-se melhores valores para as características funcionais das proteínas. Apesar de que para a diferença de cor o tratamento $\mathrm{n}^{\circ} 4$ foi o melhor. No entanto, para os parâmetros cor não foram decisivos para a determinação da melhor condição, pois para todos os tratamentos o produto final seco apresentou tonalidade amarelo-esverdeada.

\section{CONCLUSÃO}

A programação linear mostrou-se satisfatória para a otimização da formulação da pasta de vegetais enriquecida de plasma bovino, através da maximização do teor proteico. As condições utilizadas na secagem em leito de jorro foram adequadas pois não apresentaram colapso do leito.

A solubilidade proteica, a capacidade de retenção de água e o índice de solubilidade em água foram afetados pela temperatura $(90$ e $110^{\circ} \mathrm{C}$ ) ao nível de $95 \%$ de significância $(\mathrm{p}<0,05)$. O tratamento com $150 \mathrm{~mL} \mathrm{~h}^{-1} \mathrm{e}$ $90^{\circ} \mathrm{C}$ apresentou os melhores resultados quanto às propriedades funcionais das proteínas, e nessa condição, o produto final apresentou tonalidade amarelo-esverdeada com maior intensidade do que na pasta in natura.

\section{NOMENCLATURA}

$A$ - Concentração proteica $\left(\mathrm{mg} \mathrm{mL}^{-1}\right)$ $a$ - Cromaticidade (adimensional)

$\alpha$ - Ângulo Hue $\left(^{\circ}\right)$

$b$ - Cromaticidade (adimensional)

$c_{i}$ - Quantidade de carboidratos de cada

constituinte (g $\left.100 \mathrm{~g}^{-1}\right)$

$C^{*}$ - Índice de saturação (\%)

$C_{1}$ - Valor mínimo de carboidratos $(\% \mathrm{~m} / \mathrm{m})$

$\mathrm{C}_{2}$ - Valor máximo de carboidratos $(\% \mathrm{~m} / \mathrm{m})$

$C R A$ - Capacidade de Retenção de Água (\%)

Dc - Diâmetro da célula $(m)$

Di - Diâmetro do orifício de entrada (m)

$\Delta E$ - Variação da cor (adimensional)

$I S A$ - Índice de solubilidade em água (\%)

$L^{*}$ - Luminosidade (\%)

$l_{i}$ - Quantidade de lipídios de cada constituinte

$\left(\mathrm{g} 100 \mathrm{~g}^{-1}\right)$

$L_{1}$ - Valor mínimo de lipídios (\%)

$L_{2}$ - Valor máximo de lipídios (\%)

$m$ - Quantidade máxima de cada constituinte

(g)

$M_{A}$ - Massa de amostra utilizada (g)

$M_{P}$ - Massa de precipitado $(\mathrm{g})$

$M_{R E}$ - Massa de resíduo evaporado (g)

$P_{T}$ - Proteína Total (\%)

$p_{i}$ - Quantidade de proteínas de cada

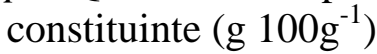

$P_{1}$ - Valor mínimo de proteínas $(\%)$

$P_{2}$ - Valor máximo de proteínas (\%)

$P S$ - Proteína Solúvel (\%)

$S$ - Concentração de proteína

$v c_{i}$ - Valor calórico de cada constituinte (kcal $\left.100 \mathrm{~g}^{-1}\right)$

$V c_{1}$ - Valor mínimo de valor calórico (kcal)

$V c_{2}$ - Valor máximo de valor calórico (kcal)

$W$ - Massa de amostra (mg)

\section{REFERÊNCIAS}

ANDERSON, R. A.; CONWAY, H. F.; PFEIFER, U. F.; GRIFFIN JR., E. L. Gelatination of corn grits by roll and extrusion cooking. Cereal Science Today, Minnesota, v. 14, n. 1, p. 4-7, 1969.

BANGA, J.; BALSA-CANTO, E.; MOLES, C.; ALONSO, A. Improving food processing 
using modern optimization methods. Trends in Food Science and Technology, v. 14, p. 131-144, 2003.

BRASIL. Ministério da Saúde - Secretaria de Vigilância Sanitária. Portaria $n^{\circ}$ 31, de 13 de janeiro de 1998. Aprova o Regulamento Técnico referente a Alimentos Adicionados de Nutrientes Essenciais, constante do anexo desta Portaria. Diário Oficial da União, Brasília, 16 janeiro de 1998a.

BRASIL. Ministério da Saúde - Secretaria de Vigilância Sanitária. Portaria $n^{\circ} 33$, de 13 de janeiro de 1998. Adota valores como níveis de IDR para as vitaminas, minerais e proteínas. Diário Oficial da União, Brasília, 16 janeiro de 1998 b.

CRISTAS, A. S. A. Capacidade de retenção de água e de gordura de diferentes concentrados proteicos usados em produtos cárneos emulsificados. 45 p. Dissertação. (Mestrado em Engenharia AlimentarQualidade e Segurança Alimentar). Instituto Superior de Agronomia-Universidade Técnica de Lisboa, Portugal. 2012.

\section{FOOD AND AGRICULTURE} ORGANIZATION. Global food losses and food waste. International Congress Save Food. Rome, 2011.

GOULART, G. A. S. Secagem de plasma bovino em leito de jorro cônico. $82 \mathrm{p}$. Dissertação. (Mestrado em Engenharia e Ciência de Alimentos). Universidade Federal do Rio Grande, Rio Grande, 2000.

INSTITUTO BRASILEIRO DE GEGRAFIA E ESTATISTICA - IBGE. Tabelas de composição de alimentos. 5 ed., p.137. Rio de Janeiro, RJ, 1999.

LARROSA, A. P. Q. Secagem de pasta de vegetais: análise da operação em leito de jorro e caracterização do produto final. 145p. Dissertação (Mestrado em Engenharia e Ciência de Alimentos) - Universidade Federal do Rio Grande, Rio Grande, 2012.

LOPES, T. J.; CANZI, C.; DUARTE FILHO, P.; HAUSEN, V. N.; PINTO, L. A. A.; FURLONG, E. B. Caracterização físicoquímica, funcional e microbiológica de sangue bovino e suas frações secas em sistema de leito de jorro. Revista Vetor, Rio Grande, v. 14, n. 1-2, p. 97-108, 2004.

MORR, C. V.; GERMAN, B.; KINSELA, J. E.; REGENSTEIN, J. M.; VAN-BUREN, J. P.; KILARA, A.; LEWIS, B. A.; MAGNINO, M. E. Collaborative study to develop a standardized food protein solubility procedure. Journal of Food Science, v. 50, p.1715-1718, 1985.

MUJUMDAR, A. S.; HUANG, L.X. Global R\&D needs in drying. Drying Technology, v. 25, p. 647-658, 2007

OLIVEIRA, H. V.A., FREITAS, L.A.P. The effect of processing factors on the efficiency of hard gelatin capsules coating in a spouted bed. In: INTERNATIONAL DRYING SYMPOSIUM, 14, 2004, São Paulo. Proceedings of the 14th International Drying Symposium. São Paulo: v. B, 2004, p. 852-859.

ORGANIZAÇÃO DAS NAÇÕES UNIDAS. Perspectivas da População Mundial: Revisão de 2012. Disponível em: < http://www.onu.org.br >. Acesso em: 23 jul. 2013.

ORNELLAS, L. H. Técnica dietética: seleção e preparo de alimento. 8 . ed. São Paulo: Atheneu Editora, 2006. 276 p. 
PRADO, D. Programação Linear. Belo

Horizonte: Editora DG, 2010. v. 1.

ROCHA, S. F.; RODRIGUES, M. C. K.; MONTE, M. L.; LARROSA, A. P. Q; PINTO, L. A. A. Product characteristics and quality of bovine blood-enriched dried vegetable paste. Journal Science Food Agriculture, v. 94, p. 3255-3262, 2014.

SGARBIERI, V. C. Proteínas em alimentos proteicos. São Paulo: Varela, 1996. 517 p.

SRINIVASA, P. C.; RAMESH, M. N.; KUMAR, K. R.; THARANATHAN, R. N. Properties of chitosan films prepared under different drying conditions. Journal of Food Engineering. v. 63, p. 79-85, 2004.

SHUHAMA, I. K.; AGUIAR, M. L.; OLIVEIRA, W. P.; FREITAS, L. A. P. Experimental production of annatto powders in spouted bed dryer. Journal of Food Engineering, v. 59, p. 93-97, 2003,

XIONG, Y. L. Dairy proteins. In. Tarté, R. Ingredients in meat products: properties, functionality and applications. New York: Springer. p. 131-143, 2009.

\section{AGRADECIMENTOS}

Os autores agradecem a Capes, ao Programa de Educação Tutorial - PET e a Universidade Federal do Rio Grande FURG. 